\title{
UNIFORM EQUICONTINUITY OF SEQUENCES OF MEASURABLE OPERATORS AND NON-COMMUTATIVE ERGODIC THEOREMS
}

\author{
SEMYON LITVINOV
}

(Communicated by Marius Junge)

\begin{abstract}
The notion of uniform equicontinuity in measure at zero for sequences of additive maps from a normed space into the space of measurable operators associated with a semifinite von Neumann algebra is discussed. It is shown that uniform equicontinuity in measure at zero on a dense subset implies the uniform equicontinuity in measure at zero on the entire space, which is then applied to derive some non-commutative ergodic theorems.
\end{abstract}

\section{INTRODUCTION AND PRELIMINARIES}

Let $(\Omega, \mu)$ be a measure space. Denote by $\mathcal{L}_{0}(\mathcal{L})$ the set of equivalence classes of all (respectively, almost everywhere finite) real or complex valued measurable functions on $\Omega$. If $X$ is a given set and $a_{n}: X \rightarrow \mathcal{L}, n=1,2, \ldots$, let

$$
a^{\star}(x)=\sup _{n}\left|a_{n}(x)\right|, x \in X .
$$

The function $a^{\star}: X \rightarrow \mathcal{L}_{0}$ is called a maximal operator associated with the family $\left\{a_{n}\right\}$.

Now let $(X,\|\cdot\|)$ be a normed space, $a_{n}: X \rightarrow \mathcal{L}$ a sequence of additive maps, and $\tau_{\mu}$ the measure topology in $\mathcal{L}$. The $\tau_{\mu}$-continuity of $a^{\star}$ at zero means that, for any given $\epsilon>0, \delta>0$, it is possible to find such $\gamma>0$ that $x \in X$ and $\|x\|<\gamma$ would imply that there exists a measurable set $E \subset \Omega$ for which the conditions

$$
\mu(\Omega \backslash E) \leq \epsilon \text { and }\left\|a^{\star}(x) \chi_{E}\right\|_{\infty} \leq \delta
$$

hold. (Here $\|\cdot\|_{\infty}$ is the uniform norm in $\mathcal{L}^{\infty}=\mathcal{L}^{\infty}(\Omega)$ and $\chi_{E}$ is the characteristic function of the set $E$.)

Proposition 1.1. If $\left\{a_{n}(x) \chi_{E}\right\}_{1}^{\infty} \subset \mathcal{L}^{\infty}$, then

$$
\left\|a^{\star}(x) \chi_{E}\right\|_{\infty}=\sup _{n}\left\|a_{n}(x) \chi_{E}\right\|_{\infty} .
$$

Proof. It is enough to show and easy to see that, given $\lambda>0, \sup _{n}\left\|a_{n}(x) \chi_{E}\right\|_{\infty}>\lambda$ if and only if $\left\|a^{\star}(x) \chi_{E}\right\|_{\infty}>\lambda$.

Therefore, $\tau_{\mu}$-continuity at zero of the maximal operator associated with a sequence of additive maps $a_{n}: X \rightarrow \mathcal{L}$ is, in fact, the uniform equicontinuity

Received by the editors February 20, 2011.

2010 Mathematics Subject Classification. Primary 46L51; Secondary 47A35.

Key words and phrases. Semifinite von Neumann algebra, uniform equicontinuity, non-commutative ergodic theorem. 
in measure of the family $\left\{a_{n}\right\}$ at zero. This will allow us to extend the notion of $\tau_{\mu}$-continuity of the maximal operator at zero to the non-commutative setting (see the definition below). The importance of this notion can be illustrated, for example, by the fact that $\tau_{\mu}$-continuity at zero of the maximal operator associated with a sequence $a_{n}: X \rightarrow \mathcal{L}$ of additive maps implies that the set $\left\{x \in X:\left\{a_{n}(x)\right\}\right.$ converges almost everywhere $\}$ is closed in $X$ (see, for instance, [4, p. 3; also 6], Theorem 2). Thus, almost everywhere convergence of the sequence $\left\{a_{n}(x)\right\}$ for every $x$ in a dense subset of $X$ entails its convergence for all $x \in X$.

Let $(H,(\cdot, \cdot))$ be a Hilbert space, $\|\cdot\|_{0}=(\cdot, \cdot)^{1 / 2}, B(H)$ the algebra of all bounded linear operators in $H$, and $\mathbb{I}$ the unit of $B(H)$. Assume that $M \subset B(H)$ is a semifinite von Neumann algebra equipped with a faithful normal semifinite trace $\tau$. We denote by $P(M)$ the complete lattice of all projections in $M$ and set $e^{\perp}=\mathbb{I}-e$ whenever $e \in P(M)$. If $\left\{e_{i}\right\}_{i \in I} \subset P(M)$, then $\bigwedge_{i \in I} e_{i}$ is the projection on the subspace $\bigcap_{i \in I} e_{i} H$.

A densely-defined closed operator $x$ in $H$ is said to be affiliated with the algebra $M$ if $x^{\prime} x \subset x x^{\prime}$ for every $x^{\prime} \in B(H)$ such that $x^{\prime} x=x x^{\prime}$ for all $x \in M$. An operator $x$ affiliated with $M$ is called $\tau$-measurable if for each $\epsilon>0$ there exists such an $e \in P(M)$ with $\tau\left(e^{\perp}\right) \leq \epsilon$ that the subspace $e H$ belongs to the domain of $x$. (In this case of course $x e \in M$.) Let $L=L(M, \tau)$ be the set of all measurable operators affiliated with the algebra $M$. Denote $\|\cdot\|_{\infty}$ as the uniform norm in $B(H)$. The topology $t_{\tau}$ defined in $L$ by the family

$\left\{V(\epsilon, \delta)=\left\{x \in L:\|x e\|_{\infty} \leq \delta\right.\right.$ for some $e \in P(M)$ with $\left.\left.\tau\left(e^{\perp}\right) \leq \epsilon\right\}: \epsilon>0, \delta>0\right\}$

of (closed) neighborhoods of zero is called a measure topology. Note that if one replaces the condition $\|x e\|_{\infty} \leq \delta$ above by the generally weaker condition $\|$ exe $\|_{\infty} \leq$ $\delta$, then the corresponding family of neighborhoods of zero generates the same topology $t_{\tau}([\underline{3}$, Theorem 2.2).

We say that a sequence $\left\{x_{n}\right\} \subset L$ converges to $x \in L$ almost uniformly (a.u.) (bilaterally almost uniformly (b.a.u.)) if for every $\epsilon>0$ there exists such an $e \in P(M)$ that $\tau\left(e^{\perp}\right) \leq \epsilon$ and $\left\|\left(x-x_{n}\right) e\right\|_{\infty} \rightarrow 0$ (respectively, $\left.\left\|e\left(x-x_{n}\right) e\right\|_{\infty} \rightarrow 0\right)$. Note that the algebra $L$ is complete with respect to both a.u. and b.a.u. convergences ([3], Theorem 2.3 and Remark 2.4).

For a positive self-adjoint operator $x=\int_{0}^{\infty} \lambda d e_{\lambda}$ affiliated with $M$ one can define

$$
\tau(x)=\sup _{n} \tau\left(\int_{0}^{n} \lambda d e_{\lambda}\right)=\int_{0}^{\infty} \lambda d \tau\left(e_{\lambda}\right) .
$$

If $1 \leq p<\infty$, then the non-commutative $L^{p}$-space associated with $(M, \tau)$ is defined as

$$
L^{p}=L^{p}(M, \tau)=\left\{x \in L:\|x\|_{p}=\left(\tau\left(|x|^{p}\right)\right)^{1 / p}<\infty\right\},
$$

where $|x|=\left(x^{*} x\right)^{1 / 2}$, the absolute value of $x$. Naturally, $L^{\infty}=M$.

In what follows, we shall use the following well-known properties of non-commutative $L^{p}$-spaces without reference:

(i) If $1 \leq p \leq \infty, x \in L^{\infty}$ and $y \in L^{p}$, then $\|x y\|_{p} \leq\|x\|_{\infty}\|y\|_{p}$.

(ii) Given $1 \leq p<\infty$, both $L^{p} \cap M$ and $L^{1} \cap M$ are dense in $L^{p}$.

(iii) If $1 \leq p \leq \infty, x, y \in L^{p}$ and $0 \leq x \leq y$, then $\|x\|_{p} \leq\|y\|_{p}$.

If $X \subset L$, we denote

$$
X_{h}=\left\{x \in X: x^{*}=x\right\} \text { and } X_{+}=\{x \in X: x \geq 0\} .
$$


The following fundamental result of Kadison [9] will be used extensively in the sequel.

Theorem 1.1 (Kadison's inequality). Let $a: M \rightarrow M$ be a positive linear map such that $a(\mathbb{I}) \leq \mathbb{I}$. Then $a(x)^{2} \leq a\left(x^{2}\right)$ for every $x \in M_{h}$.

\section{UNIFORM EQUICONTINUITY IN NON-COMMUTATIVE SETTING}

Let $X$ be any set. If $a_{i}: X \rightarrow L, i \in I$, and $e \in P(M)$ are such that $\left\{a_{i}(x) e\right\}_{i \in I} \subset$ $M\left(\left\{e a_{i}(x) e\right\}_{i \in I} \subset M\right)$, we denote

$$
a^{*}(x, e)=\sup _{i \in I}\left\|a_{i}(x) e\right\|_{\infty} \quad\left(\text { respectively, } a_{b}^{*}(x, e)=\sup _{i \in I}\left\|e a_{i}(x) e\right\|_{\infty}\right) .
$$

Definition 2.1. Let $(X,\|\cdot\|)$ be a normed space and $X_{0}$ a subset of $X$ such that $0 \in X_{0}$, where 0 is the neutral element of $X$. A family $a_{i}: X \rightarrow L, i \in I$, of additive maps is called uniformly equicontinuous in measure (u.e.m.) (bilaterally uniformly equicontinuous in measure (b.u.e.m.)) at 0 on $X_{0}$ if, given $\epsilon>0, \delta>0$, there is such a $\gamma>0$ that for every $x \in X_{0}$ with $\|x\|<\gamma$ there exists $e \in P(M)$ for which

$$
\left.\tau\left(e^{\perp}\right) \leq \epsilon \text { and } a^{*}(x, e) \leq \delta \text { (respectively, } a_{b}^{*}(x, e) \leq \delta\right) .
$$

Remark 2.1. As we explained in the previous section, in the commutative case with a countable $I$, the notion of uniform equicontinuity (bilateral uniform equicontinuity) in measure at 0 of a family $\left\{a_{i}\right\}_{i \in I}$ reduces to $\tau_{\mu}$-continuity at zero of the maximal operator associated with this family.

Lemma 2.1 ([2, Lemma 1.6]). Let $(X,+)$ be a semigroup, and let $a_{n}: X \rightarrow L$ be a sequence of additive maps. Assume that $x \in X$ is such that for every $\epsilon>0$ there exist a sequence $\left\{x_{k}\right\} \subset X$ and a projection $e \in P(M)$ such that the following conditions hold:

(i) $\left\{a_{n}\left(x+x_{k}\right)\right\}$ converges a.u. (b.a.u.) as $n \rightarrow \infty$ for each $k$;

(ii) $\tau\left(e^{\perp}\right) \leq \epsilon$;

(iii) $a^{*}\left(x_{k}, e\right) \rightarrow 0$ (respectively, $a_{b}^{*}\left(x_{k}, e\right) \rightarrow 0$ ).

Then the sequence $\left\{a_{n}(x)\right\}$ also converges a.u. (b.a.u., respectively).

Theorem 2.1 (see 2], Theorem 2.3). Assume that $(X,\|\cdot\|)$ is a normed space and $a_{n}: X \rightarrow L$ is a sequence of additive maps. If the family $\left\{a_{n}\right\}$ is u.e.m. (b.u.e.m.) at 0 on $X$, then the set $C=\left\{x \in X:\left\{a_{n}(x)\right\}\right.$ converges a.u. (respectively, b.a.u.) $\}$ is closed in $X$.

Proof. We shall consider the case of b.a.u. convergence; proof for the a.u. convergence is similar.

Fix $\epsilon>0$. Since the family $\left\{a_{n}\right\}$ is b.u.e. at zero on $X$, for every given $k \in \mathbb{N}$, there is such a $\gamma_{k}>0$ that $x \in X$ and $\|x\|<\gamma_{k}$ imply that it is possible to find $e_{k, x} \in P(M)$ for which

$$
\tau\left(e_{k, x}^{\perp}\right) \leq \frac{\epsilon}{2^{k}} \text { and } a_{b}^{*}\left(x, e_{k, x}\right) \leq \frac{1}{k} .
$$

Pick $x \in \bar{C}$. Given $k \in \mathbb{N}$, let $y_{k} \in C$ be such that $\left\|y_{k}-x\right\|<\gamma_{k}$. Then, denoting $y_{k}-x=x_{k}$, let a sequence $\left\{e_{k}\right\} \subset P(M)$ be such that

$$
\tau\left(e_{k}^{\perp}\right) \leq \frac{\epsilon}{2^{k}} \text { and } a_{b}^{*}\left(x_{k}, e_{k}\right) \leq \frac{1}{k}, k=1,2, \ldots .
$$


We have $x+x_{k}=y_{k} \in C$ for every $k$. Also, if $e=\bigwedge e_{k}$, then

$$
\tau\left(e^{\perp}\right) \leq \epsilon \text { and } a_{b}^{*}\left(x_{k}, e\right) \leq \frac{1}{k} \rightarrow 0 .
$$

Consequently, Lemma 2.1 yields $x \in C$.

Let a positive linear map $\alpha: L^{1} \rightarrow L^{1}$ be such that $\alpha(x) \leq \mathbb{I}$ and $\tau(\alpha(x)) \leq \tau(x)$ for every $x \in L^{1} \cap M$ with $0 \leq x \leq \mathbb{I}$. Note that, as it is shown in [12], $\|\alpha(x)\|_{p} \leq$ $\|x\|_{p}$ for each $x \in L_{h}^{1} \cap M$ and all $1 \leq p \leq \infty$. Besides, there exist unique continuous extensions $\alpha: L^{p} \rightarrow L^{p}$ for every $1 \leq p<\infty$ and a unique ultra-weakly continuous extension $\alpha: M \rightarrow M$.

If $x \in L^{1}$, denote

$$
a_{n}(x)=\frac{1}{n} \sum_{k=0}^{n-1} \alpha^{k}(x), n=1,2, \ldots .
$$

The following theorem due to Yeadon [12] plays an important role in this article.

Theorem 2.2. If the maps $a_{n}: L^{1} \rightarrow L^{1}$ are given by (1), then, for every $x \in L^{1}$ and $\nu>0$, there is such an $e \in P(M)$ that

$$
\tau\left(e^{\perp}\right) \leq \frac{4\|x\|_{1}}{\nu} \text { and } a_{b}^{*}(x, e) \leq 4 \nu .
$$

Remark 2.2. If $x \in L_{+}^{1}$, then $e \in P(M)$ in Theorem 2.2 can be chosen to satisfy

$$
\tau\left(e^{\perp}\right) \leq \frac{\|x\|_{1}}{\nu} \text { and } a_{b}^{*}(x, e) \leq \nu
$$

Corollary 2.1. The family $\left\{a_{n}\right\}$ given by formula $(1)$ is b.u.e.m. at 0 on $\left(L^{1},\|\cdot\|_{1}\right)$.

Corollary 2.2. If $X_{0}=L^{1} \cap\left\{x \in L^{\infty}:\|x\|_{\infty} \leq 1\right\}$, then the family $\left\{a_{n}\right\}$ given by (1) is u.e.m. at 0 on $\left(X_{0},\|\cdot\|_{1}\right)$.

Proof. Fix $\epsilon>0$ and $\delta>0$, and let $\nu>0$ and $\gamma>0$ be such that $2 \nu^{1 / 2} \leq \delta$ and $\frac{4 \gamma}{\nu} \leq \epsilon$. Take $x \in X_{0}$ with $\|x\|_{1}<\gamma$. Since $x^{2} \in L^{1}$, Theorem 2.2 implies that there exists such an $e \in P(M)$ that $\tau\left(e^{\perp}\right) \leq \frac{4\left\|x^{2}\right\|_{1}}{\nu}$ and $a_{b}^{*}\left(x^{2}, e\right) \leq 4 \nu$. Then we have $\tau\left(e^{\perp}\right)<\frac{4 \gamma}{\nu} \leq \epsilon$ and, by virtue of Kadison's inequality,

$$
\left\|a_{n}(x) e\right\|_{\infty}^{2}=\left\|e a_{n}(x)^{2} e\right\|_{\infty} \leq\left\|e a_{n}\left(x^{2}\right) e\right\|_{\infty} \leq 4 \nu \text { for every } n,
$$

implying that $a^{*}(x, e) \leq \delta$.

\section{Reduction to a Dense subset}

In this section we will show that the problem of determining whether a sequence of $t_{\tau}$-continuous additive maps is u.e.m. (b.u.e.m.) at 0 on a given set can be reduced to considering a dense subset of this set.

Lemma 3.1 ([5, Lemma 3]). Let $b \in M, 0 \leq b \leq \mathbb{I}$, and let e be the spectral projection of $b$ corresponding to the interval $[1 / 2,1]$. Then:

(i) $\tau\left(e^{\perp}\right) \leq 2 \tau(\mathbb{I}-b)$;

(ii) there exists $b^{-} \in M$ such that $\left\|b^{-}\right\|_{\infty} \leq 2$ and $e=b b^{-}$.

Proposition 3.1 ([10, see also [5], Proposition 1]). If $\left\{y_{n}\right\} \subset L$ and $y_{n} \rightarrow 0$ in $t_{\tau}$, then $y_{n_{k}} \rightarrow 0$ a.u. for some subsequence $\left\{y_{n_{k}}\right\} \subset\left\{y_{n}\right\}$. 
Theorem 3.1. Let $(X,\|\cdot\|)$ be a Banach space, and let $a_{n}: X \rightarrow\left(L, t_{\tau}\right)$ be a sequence of continuous additive maps. If the family $\left\{a_{n}\right\}$ is b.u.e.m. at 0 on a set $X_{0} \subset X$ such that $\left\{a_{n}(x)\right\} \subset L_{+}$for all $x \in \bar{X}_{0}$, where $\bar{X}_{0}$ is the closure of $X_{0}$ in $X$, then it is also b.u.e.m. at 0 on $\bar{X}_{0}$.

Proof. We employ the scheme of the proof of closedness of the sets $X_{L, k}$ in [3], Theorem 2.7. Fix $\epsilon>0$ and $\delta>0$. Since $\left\{a_{n}\right\}$ is b.u.e.m. at 0 on $X_{0}$, there exists a $\gamma>0$ such that for every $x \in X_{0}$ with $\|x\|<\gamma$ it is possible to find a projection $e_{x} \in P(M)$ satisfying

$$
\tau\left(e_{x}^{\perp}\right) \leq \frac{\epsilon}{2} \quad \text { and } \quad a_{b}^{*}\left(x, e_{x}\right)=\sup _{n}\left\|e_{x} a_{n}(x) e_{x}\right\|_{\infty} \leq \frac{\delta}{4} .
$$

Take any $x \in X_{0},\|x\|<\gamma$. As $\left|a_{n}(x)^{1 / 2} e_{x}\right|^{2}=e_{x} a_{n}(x) e_{x} \in M$, we have $\left|a_{n}(x)^{1 / 2} e_{x}\right| \in M$, hence $a_{n}(x)^{1 / 2} e_{x} \in M$, for every $n$, so we can write

$$
\sup _{n}\left\|a_{n}(x)^{1 / 2} e_{x}\right\|_{\infty}=\left[\sup _{n}\left\|e_{x} a_{n}(x) e_{x}\right\|_{\infty}\right]^{1 / 2} \leq \frac{\sqrt{\delta}}{2} .
$$

Pick $x \in \bar{X}_{0}$ such that $\|x\|<\gamma$. Then there is a sequence $\left\{y_{m}\right\} \subset X_{0}$ for which we have $\left\|x-y_{m}\right\| \rightarrow 0$ and $\left\|y_{m}\right\|<\gamma, m=1,2, \ldots$. It follows that $a_{1}\left(y_{m}\right) \rightarrow a_{1}(x)$ in $t_{\tau}$ implying $a_{1}\left(y_{m}\right)^{1 / 2} \rightarrow a_{1}(x)^{1 / 2}$ in $t_{\tau}$ [11]. Therefore, due to Proposition 3.1] $a_{1}\left(y_{m}^{(1)}\right)^{1 / 2} \rightarrow a_{1}(x)^{1 / 2}$ a.u. for some subsequence $\left\{y_{m}^{(1)}\right\} \subset\left\{y_{m}\right\}$. By the same argument, one can find a subsequence $\left\{y_{m}^{(2)}\right\} \subset\left\{y_{m}^{(1)}\right\}$ such that $a_{2}\left(y_{m}^{(2)}\right)^{1 / 2} \rightarrow$ $a_{2}(x)^{1 / 2}$ a.u. Repeating this procedure, given $n \geq 3$, we choose a subsequence $\left\{y_{m}^{(n)}\right\} \subset\left\{y_{m}^{(n-1)}\right\}$ for which the convergence $a_{n}\left(y_{m}^{(n)}\right)^{1 / 2} \rightarrow a_{n}(x)^{1 / 2}$ a.u., $m \rightarrow \infty$, takes place. If we define $x_{m}=y_{m}^{(m)}, m=1,2, \ldots$, then, taking into account that, for every $n,\left\{x_{m}\right\}_{m \geq n}$ is a subsequence of $\left\{y_{m}^{(n)}\right\}$, we conclude that

$$
a_{n}\left(x_{m}\right)^{1 / 2} \rightarrow a_{n}(x)^{1 / 2} \text { a.u. as } m \rightarrow \infty, n=1,2, \ldots .
$$

Now, because $\left\{x_{m}\right\} \subset X_{0}$ and $\left\|x_{m}\right\|<\gamma$ for all $m$, one can present a sequence $\left\{e_{m}\right\} \subset P(M)$ such that

$$
\sup _{n}\left\|a_{n}\left(x_{m}\right)^{1 / 2} e_{m}\right\|_{\infty} \leq \frac{\sqrt{\delta}}{2} \quad \text { and } \quad \tau\left(e_{m}^{\perp}\right) \leq \frac{\epsilon}{2}, \quad m=1,2, \ldots .
$$

Since the unit ball of $M$ is compact in the weak operator topology, there are a subnet $\left\{e_{\alpha}\right\} \subset\left\{e_{m}\right\}$ and $b \in M$ such that $e_{\alpha} \rightarrow b$ weakly. Then we clearly have $0 \leq b \leq \mathbb{I}$. Moreover, by the well-known inequality (see [1], for example),

$$
\tau(\mathbb{I}-b) \leq \liminf _{\alpha} \tau\left(e_{\alpha}^{\perp}\right) \leq \frac{\epsilon}{2} .
$$

Show that $\sup _{n}\left\|a_{n}(x)^{1 / 2} b\right\|_{\infty} \leq \sqrt{\delta} / 2$. For that, fix $n$ and denote $a_{n}(x)^{1 / 2}=z$ and $a_{n}\left(x_{m}\right)^{1 / 2}=z_{m}, m=1,2, \ldots$. Since $z_{m} \rightarrow z$ a.u., given $k \in \mathbb{N}$, there exists $p_{k} \in P(M)$ such that $\tau\left(p_{k}^{\perp}\right) \leq 1 / k$ and

$$
\left\|p_{k}\left(z-z_{m}\right)\right\|_{\infty}=\left\|\left(z-z_{m}\right) p_{k}\right\|_{\infty} \rightarrow 0 \text { as } m \rightarrow \infty .
$$

Let us show first that $\left\|p_{k} z b\right\|_{\infty} \leq \sqrt{\delta} / 2$ for any fixed $k$. Pick $\nu>0$ and find $m_{0}$ such that

$$
\left\|p_{k}\left(z-z_{m}\right)\right\|_{\infty}<\nu \text { whenever } m \geq m_{0}
$$


Next, fix $\xi, \eta \in H$ such that $\|\xi\|_{0}=\|\eta\|_{0}=1$. Since $e_{\alpha} \rightarrow b$ weakly, we can find such an index $\alpha(\nu)$ that

$$
\left|\left(\left(b-e_{\alpha}\right) \xi, z p_{k} \eta\right)\right|<\nu \text { for all } \alpha>\alpha(\nu) .
$$

Furthermore, because $\left\{e_{\alpha}\right\}$ is a subnet of $\left\{e_{m}\right\}$, it is possible to choose an index $\alpha\left(m_{0}\right)$ for which $\left\{e_{\alpha}\right\}_{\alpha>\alpha\left(m_{0}\right)} \subset\left\{e_{m}\right\}_{m \geq m_{0}}$. In particular, if $\alpha_{0} \geq \max \left\{\alpha(\nu), \alpha\left(m_{0}\right)\right\}$, then $e_{\alpha_{0}}=e_{m_{1}}$ for some $m_{1} \geq m_{0}$. It follows now from (2)-(4) that

$$
\begin{aligned}
\left|\left(p_{k} z b \xi, \eta\right)\right| & \leq\left|\left(p_{k} z_{m_{1}} e_{m_{1}} \xi, \eta\right)\right|+\left|\left(p_{k}\left(z-z_{m_{1}}\right) e_{m_{1}} \xi, \eta\right)\right| \\
+ & \left|\left(\left(b-e_{m_{1}}\right) \xi, z p_{k} \eta\right)\right| \leq \frac{\sqrt{\delta}}{2}+2 \nu .
\end{aligned}
$$

Remembering that $\nu>0$ was taken arbitrarily, we arrive at

$$
\left\|p_{k} z b\right\|_{\infty}=\sup _{\|\xi\|_{0}=\|\eta\|_{0}=1}\left|\left(p_{k} z b \xi, \eta\right)\right| \leq \frac{\sqrt{\delta}}{2}, k=1,2, \ldots .
$$

As $p_{k} \rightarrow \mathbb{I}$ weakly, $p_{k} z b \rightarrow z b$ weakly, and we obtain

$$
\left\|a_{n}(x)^{1 / 2} b\right\|_{\infty}=\|z b\|_{\infty} \leq \liminf _{k}\left\|p_{k} z b\right\|_{\infty} \leq \frac{\sqrt{\delta}}{2}, n=1,2, \ldots .
$$

Now let $e$ be the spectral projection of $b$ corresponding to the interval $[1 / 2,1]$. Then, by Lemma 3.1. we have $\tau\left(e^{\perp}\right) \leq \epsilon$ and

$$
\sup _{n}\left\|a_{n}(x)^{1 / 2} e\right\|_{\infty} \leq 2 \sup _{n}\left\|a_{n}(x)^{1 / 2} b\right\|_{\infty} \leq \sqrt{\delta} .
$$

Finally, we can write

$$
a_{b}^{*}(x, e)=\sup _{n}\left\|e a_{n}(x) e\right\|=\sup _{n}\left\|a_{n}(x)^{1 / 2} e\right\|_{\infty}^{2} \leq \delta ;
$$

thus, the family $\left\{a_{n}\right\}$ is b.u.e.m. at 0 on $\bar{X}_{0}$.

Repeating the procedure in the proof of Theorem 3.1 with obvious simplifications, we obtain the following.

Theorem 3.2 (cf. [7, Theorem 3.1.1). Let $X$ be a Banach space, and let $a_{n}: X \rightarrow$ $\left(L, t_{\tau}\right)$ be a sequence of continuous additive maps. If the family $\left\{a_{n}\right\}$ is u.e.m. at 0 on a set $X_{0} \subset X$, then it is also u.e.m. at 0 on the closure of $X_{0}$ in $X$.

\section{INDIVIDUAL ERGODIC THEOREMS}

Individual ergodic theorems in $L^{p}$-spaces associated with a semifinite von Neumann algebra for $1<p<\infty$ were established in [8] as an application of their maximal ergodic inequalities. Note that the settings of 8 seem to be different from that of [12]: a map $\alpha$ in [8] is initially defined on $M$, and it is assumed that $\|\alpha(x)\|_{\infty} \leq\|x\|_{\infty}$ for each $x \in M$; whereas in [12] $\alpha$ is initially defined on $L^{1}$, and it is shown that it can be uniquely extended to an ultra-weakly continuous map on $M$. In what follows we show how such ergodic theorems, in the set-up of [12, can be obtained directly from Theorem 2.2 with the help of Kadison's inequality and Theorems 3.1 and 3.2 . Everywhere in this section, unless specified differently, $1 \leq p<\infty$.

We start with the following. 
Lemma 4.1. Let $a_{n}: L^{p} \rightarrow L, n=1,2, \ldots$. Then the family $\left\{a_{n}\right\}$ is u.e.m. (b.u.e.m.) at 0 on $\left(L^{p},\|\cdot\|_{p}\right)$ if and only if it is u.e.m. (respectively, b.u.e.m.) at 0 on $\left(L_{+}^{p},\|\cdot\|_{p}\right)$.

Proof. Let us consider the bilateral case. The "only if" part is obvious. To prove the "if" part, fix $\epsilon>0$ and $\delta>0$. Then there is such a $\gamma>0$ that $x \in L_{+}^{p}$ and $\|x\|_{p}<\gamma$ imply that $a_{b}^{*}(x, e) \leq \frac{\delta}{4}$ for some $e \in P(M)$ with $\tau\left(e^{\perp}\right) \leq \frac{\epsilon}{4}$.

Take $x \in L^{p}$ such that $\|x\|_{p}<\gamma$. Then $x=x_{1}-x_{2}+i\left(x_{3}-x_{4}\right)$, where $x_{i} \in L_{+}^{p}$ and $\left\|x_{i}\right\|_{p} \leq\|x\|_{p}<\gamma, i=1,2,3,4$. Therefore, there exists $e_{i} \in P(M)$ such that $\tau\left(e_{i}^{\perp}\right) \leq \frac{\epsilon}{4}$ and $a_{b}^{*}\left(x_{i}, e_{i}\right) \leq \frac{\delta}{4}, i=1,2,3,4$. Now, if $e=\bigwedge_{i=1}^{4} e_{i}$, then $\tau\left(e^{\perp}\right) \leq \epsilon$ and

$$
a_{b}^{*}(x, e) \leq \sum_{i=1}^{4} a_{b}^{*}\left(x_{i}, e_{i}\right) \leq \delta .
$$

Thus, the assertion holds.

Assume now that the family $\left\{a_{n}\right\}$ is always given by (1).

Proposition 4.1. The family $\left\{a_{n}\right\}$ is u.e.m. at 0 on $\left(L^{2},\|\cdot\|_{2}\right)$.

Proof. Since $\overline{L_{+}^{1} \cap M} \|^{\|\cdot\|_{2}}=L_{+}^{2}$, due to Theorem 3.2 (with $X=\left(L^{2},\|\cdot\|_{2}\right)$ and $\left.X_{0}=L_{+}^{1} \cap M\right)$ and Lemma 4.1, it is enough to show that $\left\{a_{n}\right\}$ is u.e.m. at 0 on $\left(L_{+}^{1} \cap M,\|\cdot\|_{2}\right)$.

Fix $\epsilon>0$ and $\delta>0$, and let $\nu>0$ and $\gamma>0$ be such that $\nu \leq \delta^{2}$ and $\frac{\gamma}{\nu} \leq \epsilon$. Take $x \in L_{+}^{1} \cap M$ with $\|x\|_{2}<\gamma$. Since $x^{2} \in L_{+}^{1}$, Theorem 2.2 (Remark 2.2) implies that $a_{b}^{*}\left(x^{2}, e\right) \leq \nu$ for some $e \in P(M)$ with $\tau\left(e^{\perp}\right) \leq \frac{\left\|x^{2}\right\|_{1}}{\nu}$. Then we have $\tau\left(e^{\perp}\right) \leq \frac{\|x\|_{2}}{\nu}<\frac{\gamma}{\nu} \leq \epsilon$ and, by Kadison's inequality,

$$
a^{*}(x, e)^{2}=\sup _{n}\left\|a_{n}(x) e\right\|_{\infty}^{2}=\sup _{n}\left\|e a_{n}(x)^{2} e\right\|_{\infty} \leq \sup _{n}\left\|e a_{n}\left(x^{2}\right) e\right\|_{\infty} \leq \nu .
$$

Hence $a^{*}(x, e) \leq \delta$, and the proof is complete.

Theorem 4.1. For each $x \in L^{2}$, the averages (1) converge a.u. to some $\hat{x} \in L^{2}$.

Proof. Since the map $\alpha$ generates a contraction in the real Hilbert space $L_{h}^{2}=\{x \in$ $\left.L^{2}: x^{*}=x\right\}$, where $(x, y)_{\tau}=\tau(x y), x, y \in L_{h}^{2}$, it is easy to verify that the set

$$
H_{0}=\left\{x \in L_{h}^{2}: \alpha(x)=x\right\}+\left\{x-\alpha(x): x \in L_{h}^{2} \cap M\right\}
$$

is dense in $\left(L_{h}^{2},\|\cdot\|_{2}\right)$. Besides, the sequence $\left\{a_{n}(x)\right\}$ converges uniformly, hence a.u., for every $x \in H_{0}$.

Next, by Proposition 4.1, $\left\{a_{n}\right\}$ is u.e.m. at 0 on $\left(L_{h}^{2},\|\cdot\|_{2}\right)$. Therefore, Theorem 2.1 implies that the averages $a_{n}$ converge a.u. for all $x \in L_{h}^{2}$, hence for all $x \in L^{2}$. Thus, it only remains to notice that its limit $\hat{x} \in L^{2}$ (see, for example, [3], Remark 2.4 and Theorem 1.2).

Corollary 4.1. The averages (1) converge a.u. for all $x \in L^{1} \cap M$.

Now we can present an alternative ending of the proof of the main result of [12].

Theorem 4.2. [12] For each $x \in L^{1}$, the averages (1) converge b.a.u. to some $\hat{x} \in L^{1}$. 
Proof. Since the set $L^{1} \cap M$ is dense in $L^{1}$ and, by Corollary 4.1, the averages $a_{n}$ converge a.u., hence b.a.u., on this set, Corollary 2.1 and Theorem 2.1 entail that the sequence $\left\{a_{n}(x)\right\}$ converges b.a.u. for all $x \in L^{1}$. Besides, by Theorems 2.3 and 1.2 in [3], its limit $\hat{x} \in L^{1}$.

Proposition 4.2. The family $\left\{a_{n}\right\}$ is b.u.e.m. at 0 on $\left(L^{p},\|\cdot\|_{p}\right)$.

Proof. Due to Lemma 4.1 it is sufficient to show that $\left\{a_{n}\right\}$ is b.u.e.m. at 0 on $\left(L_{+}^{p},\|\cdot\|_{p}\right)$, which in turn, by Theorem 3.1, would follow from that of $\left(L_{+}^{1} \cap M,\|\cdot\|_{p}\right)$.

So, let us fix $\epsilon>0$ and $\delta>0$. There exists such a $t=t(p, \delta)$ that $t \cdot \lambda^{p} \geq \lambda$ whenever $\lambda \geq \frac{\delta}{2}$. Let $\nu>0$ and $\gamma>0$ be such that $\nu \leq \frac{\delta}{2 t}$ and $\frac{\gamma}{\nu} \leq \epsilon$.

Take $x \in L_{+}^{1} \cap M$ such that $\|x\|_{p}<\gamma$. We have

$$
x=\int_{0}^{\|x\|_{\infty}} \lambda d e_{\lambda}=\int_{0}^{\delta / 2} \lambda d e_{\lambda}+\int_{\delta / 2}^{\|x\|_{\infty}} \lambda d e_{\lambda} \leq x_{\delta}+t \int_{\delta / 2}^{\|x\|_{\infty}} \lambda^{p} d e_{\lambda} \leq x_{\delta}+t \cdot x^{p},
$$

where $x_{\delta}=\int_{0}^{\delta / 2} \lambda d e_{\lambda}$, so that $\left\|x_{\delta}\right\|_{\infty} \leq \frac{\delta}{2}$ and $0 \leq x_{\delta} \leq x$, implying that $x_{\delta} \in$ $L_{+}^{1} \cap M$, which in turn entails that $\left\|a_{n}\left(x_{\delta}\right)\right\|_{\infty} \leq\left\|x_{\delta}\right\|_{\infty} \leq \frac{\delta}{2}$ for all $n$.

Next, since $x \in L_{+}^{p}$, it follows that $x^{p} \in L_{+}^{1}$. Therefore, by Theorem 2.2 (Remark 2.2), one can find such an $e \in P(M)$ that $\tau\left(e^{\perp}\right) \leq \frac{\left\|x^{p}\right\|_{1}}{\nu}=\frac{\|x\|_{p}}{\nu}<\frac{\gamma}{\nu} \leq \epsilon$ and $a_{b}^{*}\left(x^{p}, e\right) \leq \nu \leq \frac{\delta}{2 t}$. Then we have

$$
a_{b}^{*}(x, e) \leq a_{b}^{*}\left(x_{\delta}, e\right)+t \cdot a_{b}^{*}\left(x^{p}, e\right) \leq \sup _{n}\left\|a_{n}\left(x_{\delta}\right)\right\|_{\infty}+\frac{\delta}{2} \leq \delta,
$$

which completes the proof.

Employing Proposition 4.2 and repeating the argument of Theorem 4.2, we derive the following.

Theorem 4.3. For each $x \in L^{p}$, the averages (1) converge b.a.u. to some $\hat{x} \in L^{p}$.

Proposition 4.3. Let $2<p<\infty$. Then the family $\left\{a_{n}\right\}$ is u.e.m. at 0 on $\left(L^{p},\|\cdot\|_{p}\right)$.

Proof. By Lemma 4.1 and Theorem 3.2 it is enough to show that $\left\{a_{n}\right\}$ is u.e.m. at 0 on $\left(L_{+}^{p} \cap M,\|\cdot\|_{p}\right)$.

Fix $\epsilon>0$ and $\delta>0$. Since, due to Proposition 4.2, $\left\{a_{n}\right\}$ is b.u.e.m. at 0 on $\left(L^{\frac{p}{2}},\|\cdot\|_{\frac{p}{2}}\right)$, there exists such a $\gamma>0$ that $y \in L^{\frac{p}{2}}$ and $\|y\|_{\frac{p}{2}}<\gamma$ imply that $a_{b}^{*}(y, e) \leq \delta^{2}$ for some $e \in P(M)$ with $\tau\left(e^{\perp}\right) \leq \epsilon$.

If one takes $x \in L_{+}^{p} \cap M$ with $\|x\|_{p}<\gamma^{1 / 2}$, then $x^{2} \in L^{\frac{p}{2}}$ and $\left\|x^{2}\right\|_{\frac{p}{2}}=\|x\|_{p}^{2}<\gamma$. Therefore, there is $e \in P(M)$ such that $\tau\left(e^{\perp}\right) \leq \epsilon$ and $a_{b}^{*}\left(x^{2}, e\right) \leq \delta^{2}$, and, applying Kadison's inequality again, we arrive at $a^{*}(x, e) \leq \delta$.

Theorem 4.4. If $2 \leq p<\infty$, then, for every $x \in L^{p}$, the averages (1) converge a.u. to some $\hat{x} \in L^{p}$.

Proof. Since the set $L^{1} \cap M$ is dense in $L^{p}$ and, by Corollary 4.1, the sequence $\left\{a_{n}(x)\right\}$ converges a.u. for every $x \in L^{1} \cap M$, applying Proposition 4.3 and then Theorem 2.1. we infer that the averages $a_{n}$ converge a.u. for all $x \in L^{p}$. The fact that the corresponding limits $\hat{x}$ belong to $L^{p}$ follows as in Theorem 4.2 . 


\section{REFERENCES}

[1] O. Bratelli, D.N. Robinson, Operator Algebras and Quantum Statistical Mechanics, Springer, Berlin, 1979.

[2] V. Chilin, S. Litvinov, Uniform equicontinuity for sequences of homomorphisms into the ring of measurable operators, Methods of Funct. Anal. Top., 12 (2)(2006), 124-130. MR2238034 (2007b:46112)

[3] V. Chilin, S. Litvinov, A. Skalski, A few remarks in non-commutative ergodic theory, J. Operator Theory, 53 (2)(2005), 331-350. MR2153152 (2006c:46053)

[4] A. Garsia, Topics in Almost Everywhere Convergence, Markham: Lectures in Advanced Mathematics, 4, 1970. MR0261253(41:5869)

[5] M. Goldstein, S. Litvinov, Banach principle in the space of $\tau$-measurable operators, Studia Math., 143(2000), 33-41. MR1814479 (2002b:46107)

[6] S. Litvinov, The Banach principle for topological groups, Atti Sem. Mat. Fis. Univ. Modena e Reggio Emilia, 53 (2005), 323-330. MR2289403 (2007j:22004)

[7] M. de Guzman, Real Variable Methods in Fourier Analysis, North-Holland: Math. Studies, 46, 1981. MR596037 (83j:42019)

[8] M. Junge, Q. Xu, Noncommutative maximal ergodic theorems, J. Amer. Math. Soc., 20 (2) (2007), 385-439. MR2276775 (2007k:46109)

[9] R. V. Kadison, A generalized Schwarz inequality and algebraic invariants for operator algebras, Ann. of Math. (2), 56(1952), 494-503. MR0051442 (14:481c)

[10] I. Segal, A non-commutative extension of abstract integration, Ann. of Math. (2), 57(1953), 401-457. MR0054864(14:991f)

[11] O.E. Tichonov, Continuity of operator functions in topologies connected with a trace on a von Neumann algebra [Russian], Izv. Vyssh. Uchebn. Zaved. Mat., 1(1987), 77-79. MR892008 (88h:46120)

[12] F. J. Yeadon, Ergodic theorems for semifinite von Neumann algebras. I, J. London Math. Soc., 16 (2)(1977), 326-332. MR0487482(58:7111)

Department of Mathematics, Pennsylvania State University, 76 University Drive, Hazleton, Pennsylvania 18202

E-mail address: snl2@psu.edu 